Check for updates

Cite this: RSC Chem. Biol., 2021, 2, 1274

Received 21st April 2021,

Accepted 24th June 2021

DOI: $10.1039 / \mathrm{d} 1 \mathrm{cb} 00087 \mathrm{j}$

rsc.li/rsc-chembio

\title{
Entropy of stapled peptide inhibitors in free state is the major contributor to the improvement of binding affinity with the GK domain $\dagger$
}

\author{
Ilona Christy Unarta, $\ddagger^{\mathrm{ab}}$ Jianchao Xu, $\ddagger^{\mathrm{c}}$ Yuan Shang, (D) $\ddagger^{\mathrm{bd}}$ Carina Hey Pui Cheung, ${ }^{\mathrm{c}}$ \\ Ruichi Zhu, bd Xudong Chen, ${ }^{\text {bd }}$ Siqin Cao, be Peter Pak-Hang Cheung, bc \\ Donald Bierer, ${ }^{\dagger}$ Mingjie Zhang, ${ }^{* b d}$ Xuhui Huang (D) *abeg and Xuechen Li (D)*c
}

\begin{abstract}
Stapled peptides are promising protein-protein interaction (PPI) inhibitors that can increase the binding potency. Different from small-molecule inhibitors in which the binding mainly depends on energetic interactions with their protein targets, the binding of stapled peptides has long been suggested to be benefited from entropy. However, it remains challenging to reveal the molecular features that lead to this entropy gain, which could originate from the stabilization of the stapled peptide in solution or from the increased flexibility of the complex upon binding. This hinders the rational design of stapled peptides as PPI inhibitors. Using the guanylate kinase (GK) domain of the postsynaptic density protein 95 (PSD-95) as the target, we quantified the enthalpic and entropic contributions by combining isothermal titration calorimetry (ITC), X-ray crystallography, and free energy calculations based on all-atom molecular dynamics (MD) simulations. We successfully designed a stapled peptide inhibitor (staple 1) of the PSD-95 GK domain that led to a 25 -fold increase in the binding affinity (from tens of $\mu$ Ms to $1.36 \mu \mathrm{M}$ ) with high cell permeability. We showed that entropy indeed greatly enhanced the binding affinity and the entropy gain was mainly due to the constrained-helix structure of the stapled peptide in solution (free state). Based on staple 1, we further designed two other stapled peptides (staple 2 and 3), which exerted even larger entropy gains compared to staple 1 because of their more flexible bound complexes (bound state). However, for staple 2 and 3, the overall binding affinities were not improved, as the loose binding in their bound states led to an enthalpic loss that largely compensated the excess entropy gain. Our work suggests that increasing the stability of the stapled peptide in free solution is an effective strategy for the rational design of stapled peptides as PPI inhibitors.
\end{abstract}

\section{Introduction}

${ }^{a}$ Bioengineering Graduate Program, Department of Biological and Chemical Engineering, The Hong Kong University of Science and Technology,

Clear Water Bay, Kowloon, Hong Kong. E-mail: xuhuihuang@ust.hk

${ }^{b}$ Centre of Systems Biology and Human Health, State Key Laboratory of Molecular Neuroscience, The Hong Kong University of Science and Technology,

Clear Water Bay, Kowloon, Hong Kong. E-mail: mzhang@ust.hk

${ }^{c}$ Department of Chemistry, State Key Lab of Synthetic Chemistry,

The University of Hong Kong, Hong Kong. E-mail: xuechenl@hku.hk

${ }^{d}$ Division of Life Science, The Hong Kong University of Science and Technology, Kowloon, Hong Kong

${ }^{e}$ Department of Chemistry, The Hong Kong University of Science and Technology, Kowloon, Hong Kong

${ }^{f}$ Department of Medicinal Chemistry, Bayer AG, Aprather Weg 18A, 42096 Wuppertal, Germany

${ }^{g}$ Hong Kong Center for Neurodegenerative Diseases, Hong Kong Science Park, Hong Kong

$\dagger$ Electronic supplementary information (ESI) available. See DOI: 10.1039/ d1cb00087j

\$ These authors contributed equally to this work.
Protein-protein interaction (PPI) facilitates many vital biological processes. Hence, it is of great importance to be able to target PPI for functional studies and drug development. Unfortunately, targeting PPI especially by using small chemical molecules is challenging because the interface of PPI is shallow but highly complex. ${ }^{1}$ Peptides represent a significant advancement in the design of inhibitors targeting PPI because they can be easily customized by simple modification of the residues to increase the specificity and biological potency of binding at the PPI interface. However, natural peptide ligands are prone to protease degradation, often have low cell permeability and bioavailability, and are unstable in the cellular environment. ${ }^{2}$ Additionally, peptides may adopt multiple conformations in solution, resulting in non-specific binding. Thus, to circumvent these limitations, stabilization of peptides in the bioactive states using methods such as peptide stapling has emerged to the forefront of peptide design. 
Peptide stapling is most commonly performed by tethering the side chains at $i$ and $i+3, i$ and $i+4$ or $i$ and $i+7$ residues via a chemical linker to chemically restrict the flexibility and maintain the $\alpha$-helix structures of the short helical peptides in water. ${ }^{3}$ Stapled peptides have become a promising class of PPI inhibitors that have been reported to improve the cell permeability, protease resistance and biological potency. ${ }^{4-7}$ Most stapled peptides have also been shown to decrease the entropy penalty $(-T \Delta S)$, leading to improvement in their binding affinity $\left(\Delta G_{\text {binding }}=\Delta H-T \Delta S\right){ }^{8}$ Experimental techniques, such as ITC measurement and surface plasmon resonance, have been applied to measure the enthalpy and entropy differences $(\Delta H$ and $\Delta S)$ of the binding free energy. However, these thermodynamic measurements lack molecular interpretations, which hinders the rational design of stapled peptide PPI inhibitors. For example, it is well-known that stapled peptides can reduce the entropy penalty $\left(-T \Delta S=-T\left(\Delta S_{\text {bound }}-S_{\text {free }}\right)\right),{ }^{8}$ which could be the result of a decrease of $S_{\text {free }}$ and/or increase of $S_{\text {bound }}$. Unfortunately, there is a lack of experimental techniques that can quantify the conformational ensemble of stapled peptides in the free state (separated from the bound state) to obtain this entropy (absolute entropy in the free state, $\left.S_{\text {free}}\right)$. Lastly, it is even more challenging to identify the major molecular features influencing the thermodynamic contributions of stapled peptides to PPI binding. Molecular dynamics (MD) simulation is an ideal technique to address the above-mentioned challenges, as it can provide atomistic details of both the bound and free states. Ultimately, it will allow for the evaluation of individual thermodynamic contributions, which will help in correlating the molecular features with the specific thermodynamics terms, further guiding the rational design of stapled peptide PPI inhibitors. ${ }^{9}$

The PPI between postsynaptic density protein 95 (PSD-95) and the SAPAP/Shank complex serves as a great system for the development of stapled peptide PPI inhibitors. PSD-95 is a member of the DLG subfamily MAGUK scaffold. ${ }^{10}$ It forms a complex with SAPAP and Shank family proteins, ${ }^{11-13}$ and this complex has been shown to play a crucial role in synaptic development and transmission. ${ }^{14-17}$ During complex formation, the guanylate kinase (GK) domain of PSD-95 (referred to as GK domain hereinafter) functions as a phosphoprotein-binding module $\mathrm{e}^{18-20}$ to recognize the SAPAP GK binding repeat (GBR) region. ${ }^{11,21}$ Previously, synthetic phosphor-peptides have been developed to block the PPI between the GK domain and SAPAP. ${ }^{22}$ However, phosphor-peptides have been shown to exhibit low cell penetrability $^{23,24}$ and cellular instability. To resolve these problems, the unstable phosphor group has been replaced with glutamic acid to maintain the salt-bridge interactions between the phosphor group and its binding pocket. Unfortunately, this resulted in an order of magnitude decrease in binding affinity. ${ }^{22}$ Importantly, these synthetic phosphor-peptides adopt the $\alpha$-helical conformation in the bound complex, and thus this system serves as an ideal target for the development of stapled peptide PPI inhibitors.

In the present work, we have successfully designed a hydrophilic staple peptide (staple 1) using our recently developed synthetic strategies ${ }^{25}$ to inhibit the GK domain with a 25 -fold stronger binding affinity compared to that of the non-stapled (linear) peptide, accompanied by a high penetrability to mammalian cells. Using a combination of X-ray crystallography, ITC measurement and free energy calculations based on MD simulations, we further elucidated that it is the stabilization of the stapled peptide in the $\alpha$-helical structure in the free state that greatly improves the binding of the stapled peptide. We further explored two additional staple peptides (staple $2 \& 3$ ). The ITC and MD simulations showed that staple $2 \& 3$ exhibited reduced entropy penalty to a larger extent than staple 1 . Further investigations show that this excess entropy gain is mainly due to the increased flexibility of the bound complex, and this gain in entropy term is accompanied by the loss of enthalpy. As a result, the overall binding affinities of staple 2 and 3 are compared with that of staple 1. Our work elucidates the molecular mechanisms for the interplay between entropic and enthalpic contributions to the binding affinity of staple peptides for PPI inhibition.

\section{Results and discussion}

\section{Optimization of stapling positions and peptide sequence}

Replacement of phosphoserine with a phosphor-mimic residue, glutamic acid, in the linear natural ligand (p-SAPAP1) diminishes the binding with the GK domain (ESI, $\dagger$ Text). To improve the binding affinity, we propose the introduction of staple to the linear peptide, which can restrain the peptide in the $\alpha$-helical structure mimicking the p-SAPAP1 ligand observed in the crystal structure of the GK domain bound with p-SAPAP1. ${ }^{22}$ First, we determined the position of the staple based on the crystal structure of the GK domain in complex with a phosphor linear peptide (phosphor-LGN, PDB ID: $\left.3 \mathrm{UAT}^{18}\right)$. We observed that three residues, at positions $-1,2$,

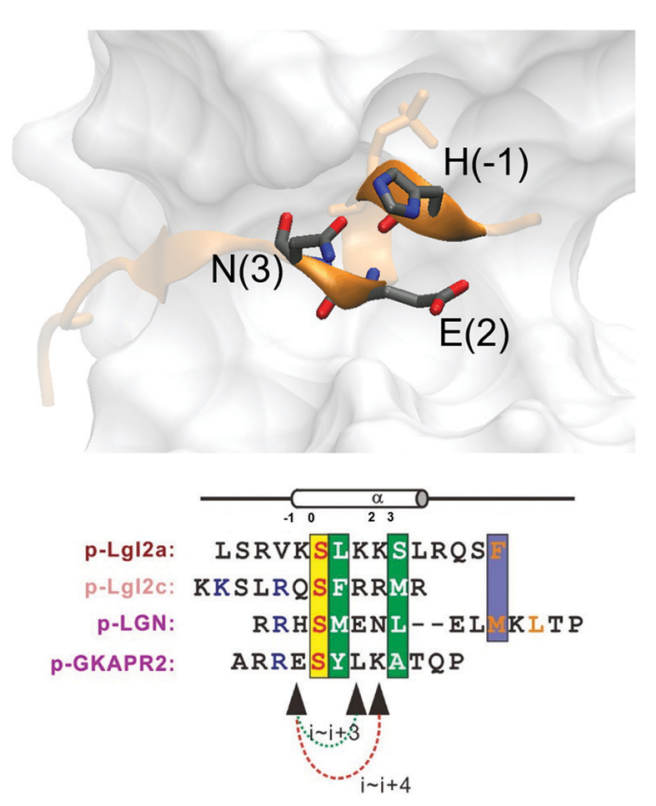

Fig. 1 (a) MAGUK GK targets form helices in the complex. Positions ( -1$)$, (2) and (3) are not directly involved in complex binding, providing opportunity for stapling. 


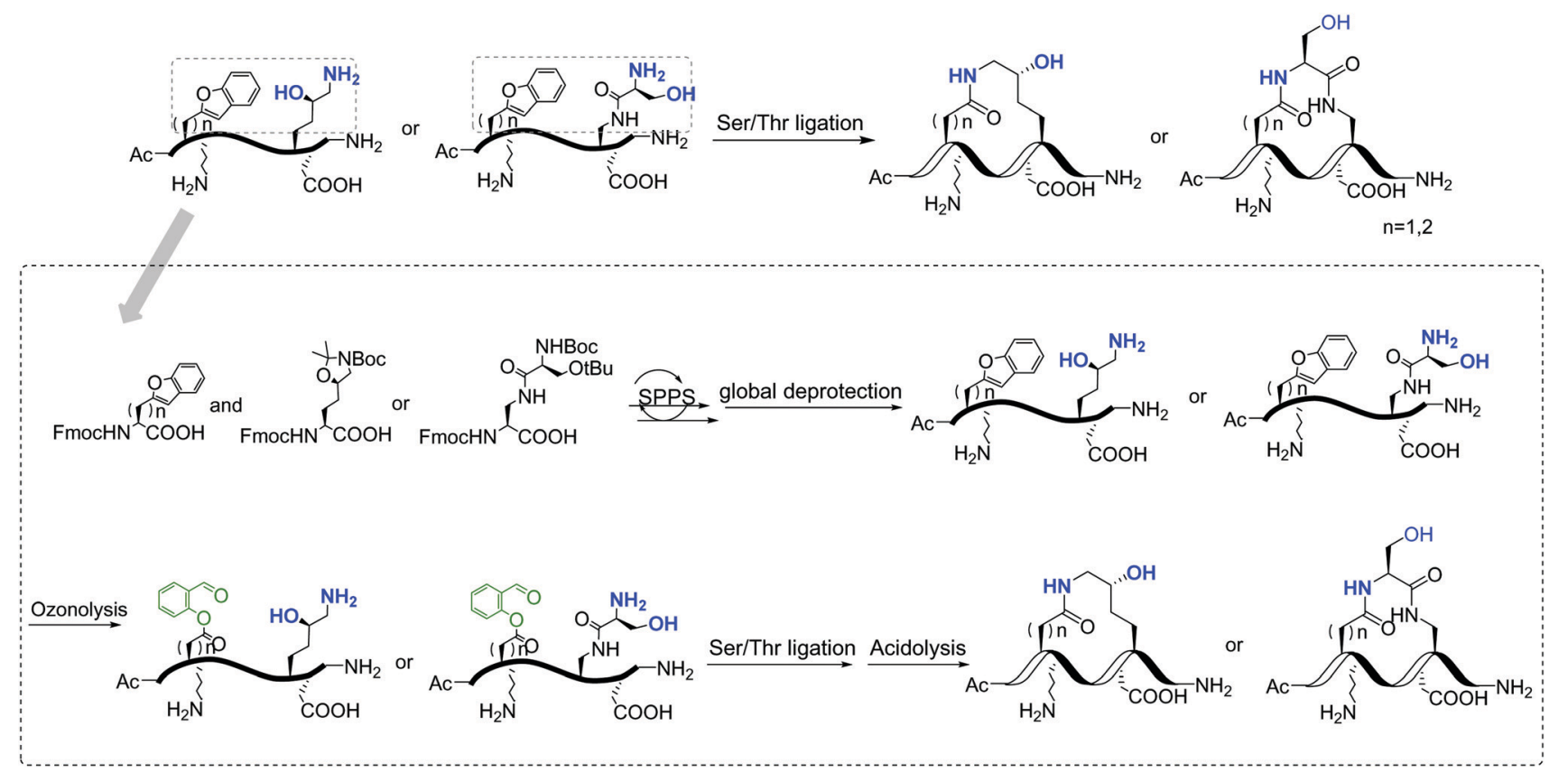

Fig. 2 General synthetic route of the involved stapled peptides. Detailed synthetic pathways of staple 1, staple 2, and staple 3 are shown in Fig. S2, ESI. $\dagger$

and 3, close to the phosphoserine (position 0 ) are facing away from the binding interface and are exposed to the solvent (Fig. 1). Therefore, we introduced our staple, either at $i \sim i+3$ (stapling at residues -1 and 2 ) or at $i \sim i+4$ (stapling at residues -1 and 3 ) to ensure that the staple is exposed to the solvent, avoiding any possible interference with the binding interface. Strikingly, the $i$ $\sim i+4$ stapled peptide displayed detectable binding to the GK domain with $K_{\mathrm{d}}=5.9 \mu \mathrm{M}$, while the $i \sim i+3$ stapled peptide behaved similarly to the linear peptide and did not bind to the GK domain (see Fig. S1, ESI $\dagger$ ). We further performed several rounds of optimizations on the linear peptide sequence based on the binding surface of GK based on previously solved crystal structures $^{18,19,22}$ (see ESI, $\dagger$ Section 2 for details). Upon these optimizations, we adopt the sequence of RIRREEYLKAIQ for further development of the stapled peptide. Our initial attempts to introduce the hydrocarbon linkers at $i \sim i+4$ positions were not successful, as they display poor solubility in aqueous solutions. To resolve this solubility issue, we designed our stapled peptide (staple 1) using our recently developed hydrophilic D-K(OH $)^{25}$ lactam stapler (Fig. 2 and Fig. S2A and B, ESI $\dagger$ ).

\section{Stapled peptide improves the binding affinity by 25 -fold by the} significant reduction of entropy penalty

As shown in Fig. 3, staple 1 peptide greatly improved the binding affinity ( $\Delta G_{\text {binding }}$ ) by 25 fold to reach $1.36 \mu \mathrm{M}$. Furthermore, to elucidate the binding modes of the GK domain with both stapled and linear peptides, we solved the crystal structure of GK-linear and GK-staple 1 peptide complexes (Fig. 4). As shown in Fig. S4A and B (ESI $\dagger$ ), the GK-linear peptide and GK-staple 1 peptide were crystallized in a similar binding pose with all the conserved binding interactions preserved. These interactions are similar in both GK-linear peptide and GK-staple 1 peptide complexes, suggesting that the enthalpy terms of both complexes are comparable and the

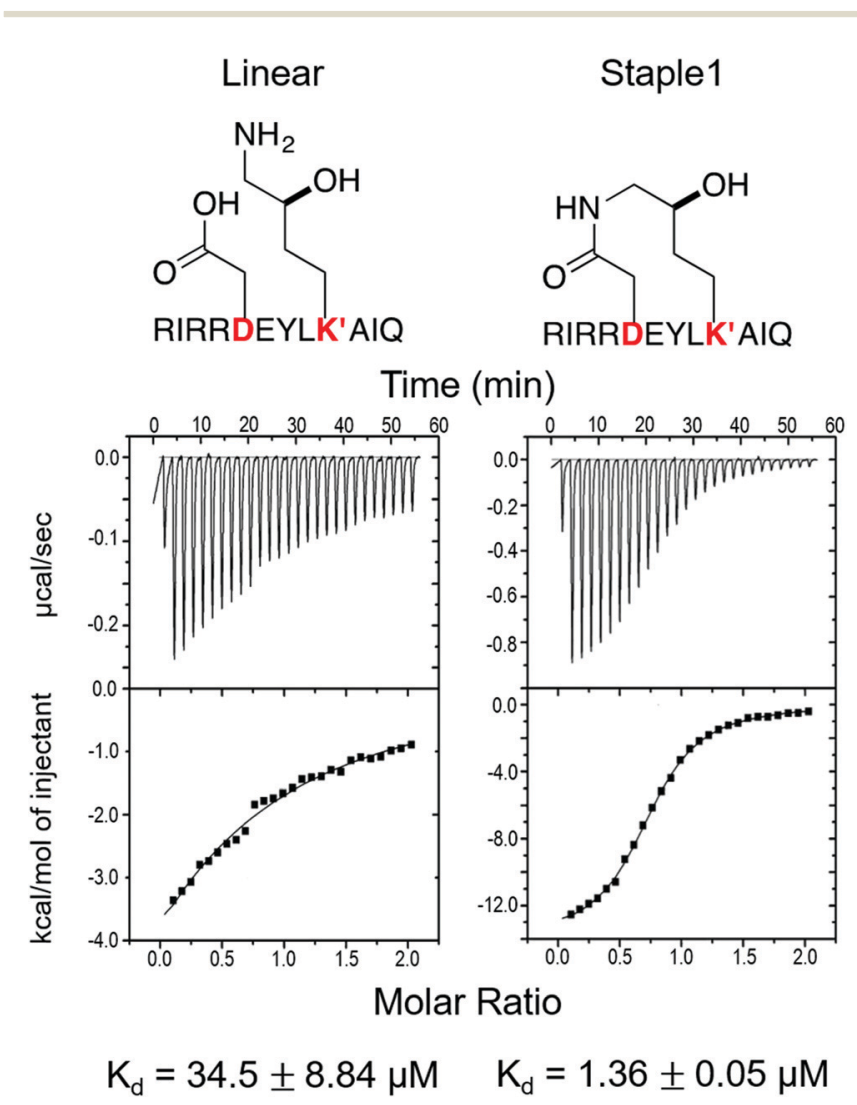

Fig. 3 Functional groups of staples and ITC-based measurements showing the binding between the PSD95 GK domain and (A) linear and (B) staple 1 peptides. 
A

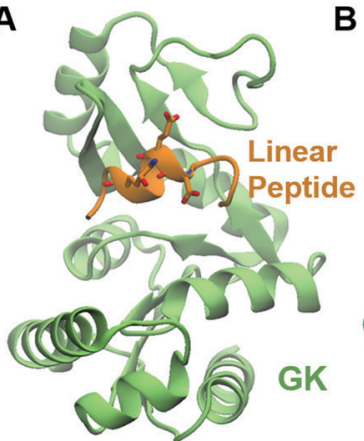

B

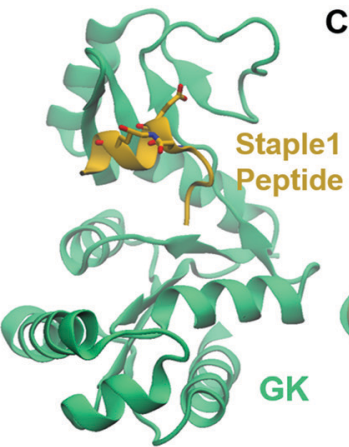

C

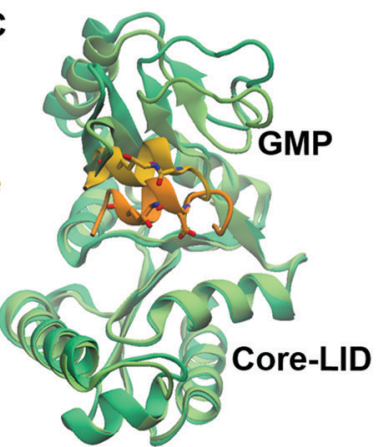

Fig. 4 Crystal structure of GK in complex with stapled peptide. Overall structure of the PSD95 GK domain in complex with (A) linear peptide and (B) staple 1 peptide. (C) Overlay of crystal structures of GK in complex with linear and staple 1 peptides aligning at the Core-LID domain.

improvement by one order of magnitude is entropy-driven. To further investigate the thermodynamic contributions to the enhancement of binding affinity of stapled peptides to the GK domain, we examined the entropic and enthalpic contributions using ITC measurements in combination with free energy calculations based on MD simulations.

Our results show that the increase in binding affinity of the staple 1 peptide is due to the reduction in entropy penalty $(-T \Delta S)$. The GK domain in complex with the staple 1 peptide has smaller entropy penalty, yet less favorable enthalpy contribution as derived by ITC-based binding experiments (Fig. 5B and C, gray bar). Specifically, the entropy term of the staple 1 peptide binding to the GK domain is stabilized by $-4.5 \mathrm{kcal} \mathrm{mol}^{-1}$ and the enthalpy term is destabilized by $2.6 \mathrm{kcal} \mathrm{mol}^{-1}$ compared with the linear peptide (Table S1, ESI $\dagger$ ). Nevertheless, the stabilization due to the entropy term

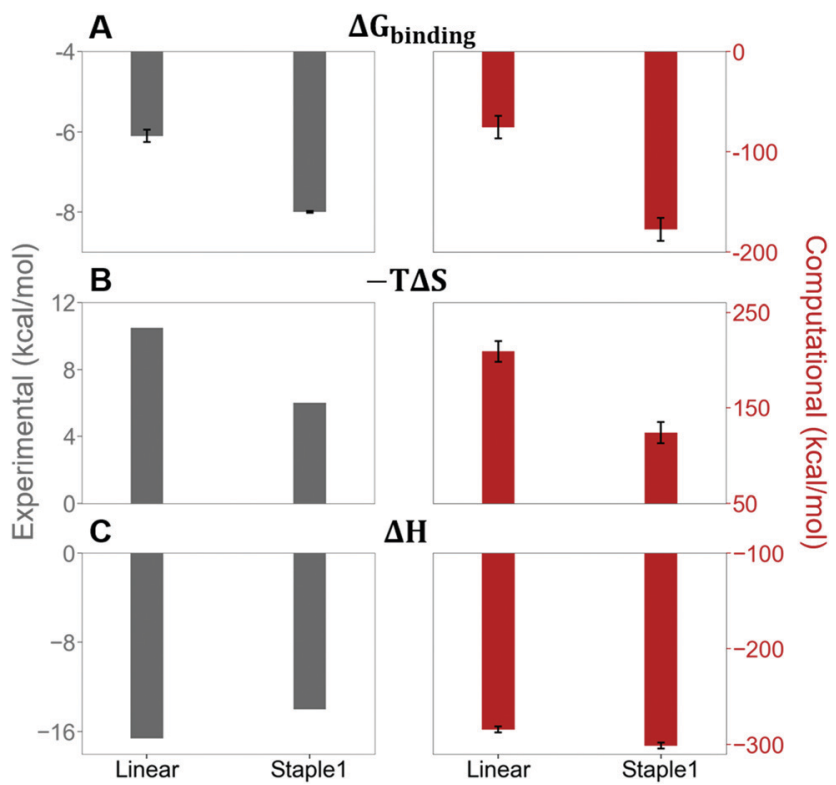

Fig. 5 Thermodynamic properties of GK binding with linear and staple 1 peptides. (A) Binding free energy $\left(\Delta G_{\text {binding }}\right)$, (B) entropy difference $(-T \Delta S)$, and $(C)$ enthalpy difference $(\Delta H)$ as measured by ITC (gray bar and left figures) and calculated by MM/PBSA and Quasi-harmonic approximation (red bar and right figures). significantly overrides the destabilization by the enthalpy term $(|-T \Delta \Delta S|>|\Delta \Delta H|)$, resulting in an order of magnitude improvement of $K_{\mathrm{d}}$ for the staple 1 peptide binding with the GK domain.

\section{Constrained stapled peptide in the free state is the main contributor to the reduction of entropy penalty}

Using conformations from MD simulations, we further computed the binding free energy using a MM/PBSA and Quasi-Harmonic approximation. Our calculation results are in reasonable agreement with experimental observations within standard deviations for all thermodynamic terms: $\Delta G_{\text {binding, }}-T \Delta S$, and $\Delta H$ (Fig. 5). Furthermore, we demonstrate that the improvement in the binding affinity of the GK domain to the staple 1 peptide is mainly due to the decrease in flexibility of the staple 1 peptide in free solution $\left(\left(S_{\text {free }}\right)_{\text {ligand }}\right.$ in Fig. 6A). Consistent with the small value of $\left(S_{\text {free }}\right)_{\text {ligand }}$, the staple 1 peptide has higher $\alpha$-helical content according to both MD simulations and the CD Spectrum (Fig. 7).

$$
\begin{gathered}
\Delta S_{\text {total }}=\Delta S_{\text {ligand }}+\Delta S_{\text {protein }} \\
\Delta S_{\text {ligand }}=\left(S_{\text {bound }}-S_{\text {free }}\right)_{\text {ligand }} \quad \Delta S_{\text {protein }}=\left(S_{\text {bound }}-S_{\text {free }}\right)_{\text {protein }} \\
\text { Absolute Entropy of Ligand }
\end{gathered}
$$

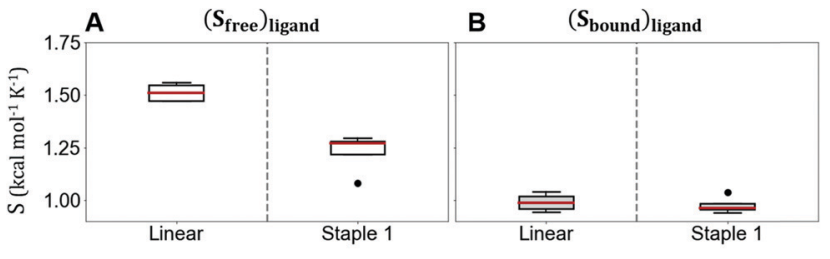

Absolute Entropy of Protein

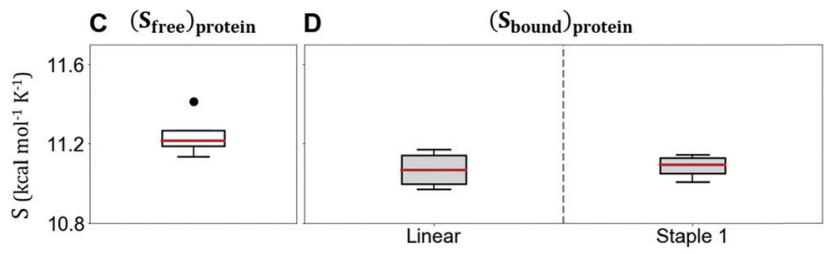

Fig. 6 Absolute entropy calculation by quasi-harmonic approximation of (A) free state, (B) bound state of linear and staple 1 peptides, (C) free state of protein, and (D) protein when bound with linear and staple 1 peptides. Equation for the calculation of computational $\Delta S_{\text {total }}$ is shown at the top. 
A
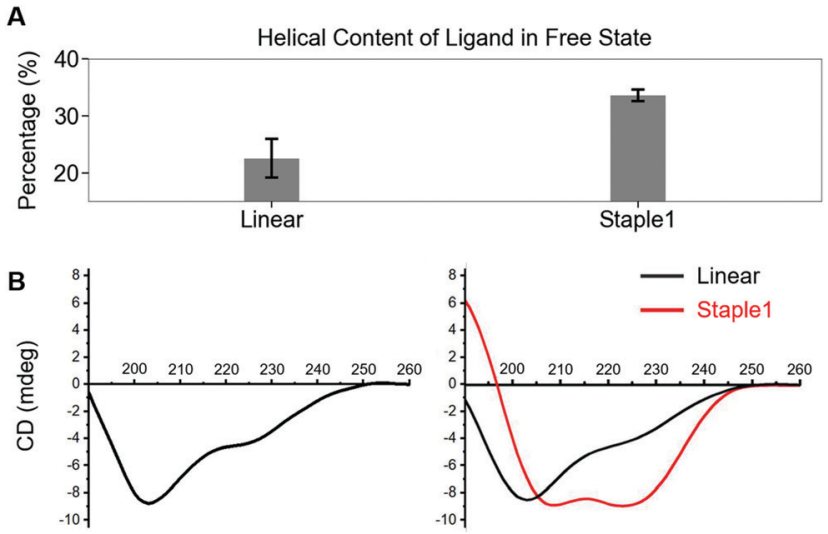

Wavelength $(\mathrm{nm})$

C
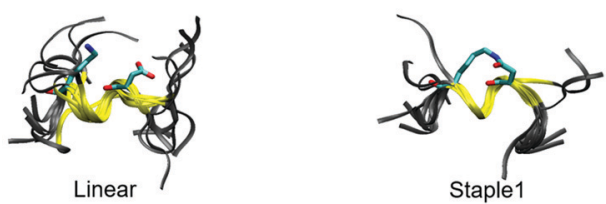

Fig. 7 Helical content calculated from MD simulation (top), CD spectrum (middle) and overlaid structures from MD simulation (bottom) of (A) linear and (B) staple 1 ligands in the free state.

In sharp contrast, the linear peptide has lower $\alpha$-helical content and is more flexible in the free state, leading to a substantially larger value of $\left(S_{\text {free }}\right)_{\text {ligand }}$ (Fig. 7). These observations clearly indicate that the rigidification in the $\alpha$-helix structure upon the introduction of the staple can significantly reduce the absolute entropy of the free peptide, resulting in much improved stabilization of binding to the GK domain. Interestingly, our calculations provide largely similar values for the absolute entropy of the bound state $\left(S_{\text {bound }}\right)$ for both the protein and ligand (Fig. 6B and D), suggesting that there is no significant contribution from the bound state to the stabilization of binding affinity of the GK-staple 1 peptide complex.

Furthermore, we found that the GK domain has less stable interaction energy with the staple 1 peptide compared with the linear peptide $\left(\Delta E_{\text {Interaction }}\right.$ in Table S3, ESI $\left.\dagger\right)$. Further analysis based on their crystal structures (Fig. 4C) and MD simulations (Fig. S5, ESI $\dagger$ ) shows that the GK domain adopts a more open conformation when bound to the staple 1 peptide compared to the linear peptide. As shown in Fig. S6B (ESI $\dagger$ ), the introduction of staple constrains the peptide in an $\alpha$-helical conformation with a larger $\alpha$-helix radius (Fig. S6B, ESI $\dagger$ ). We speculate that this may subsequently induce the opening of the GK domain, leading to less stable interaction and eventually less favorable enthalpy between the GK domain and the stapled peptide than with the linear peptide.

\section{Stapled peptide designs benefitting from increased bound state entropy could not improve the overall binding affinities due to the energy-entropy compensation}

In this section, we synthesize two more stapled peptides, staple 2 and staple 3 peptides (Fig. 8A and Fig. S2C, ESI $\dagger$ ). As shown in Fig. $8 \mathrm{C}$ and D, the experimental and free energy calculation results showed that the entropy penalty for the binding of staple 2 and 3 peptides is further reduced compared to the staple 1 peptide. Surprisingly, these two stapled peptides do not improve the overall binding affinity compared to the staple 1 peptide (Fig. 8). Further analysis reveals that the further reduction of the entropy penalty in staple 2 and 3 peptides is not due to $\left(S_{\text {free }}\right)_{\text {ligand }}$ but due to the increase of the GK domain entropy in the bound state, $\left(S_{\text {bound }}\right)_{\text {protein }}$ (Fig. S7, ESI $\dagger$ ). This increase in the flexibility of the GK domain in the bound state reflects a flexible binding mode with its ligand. Indeed, our
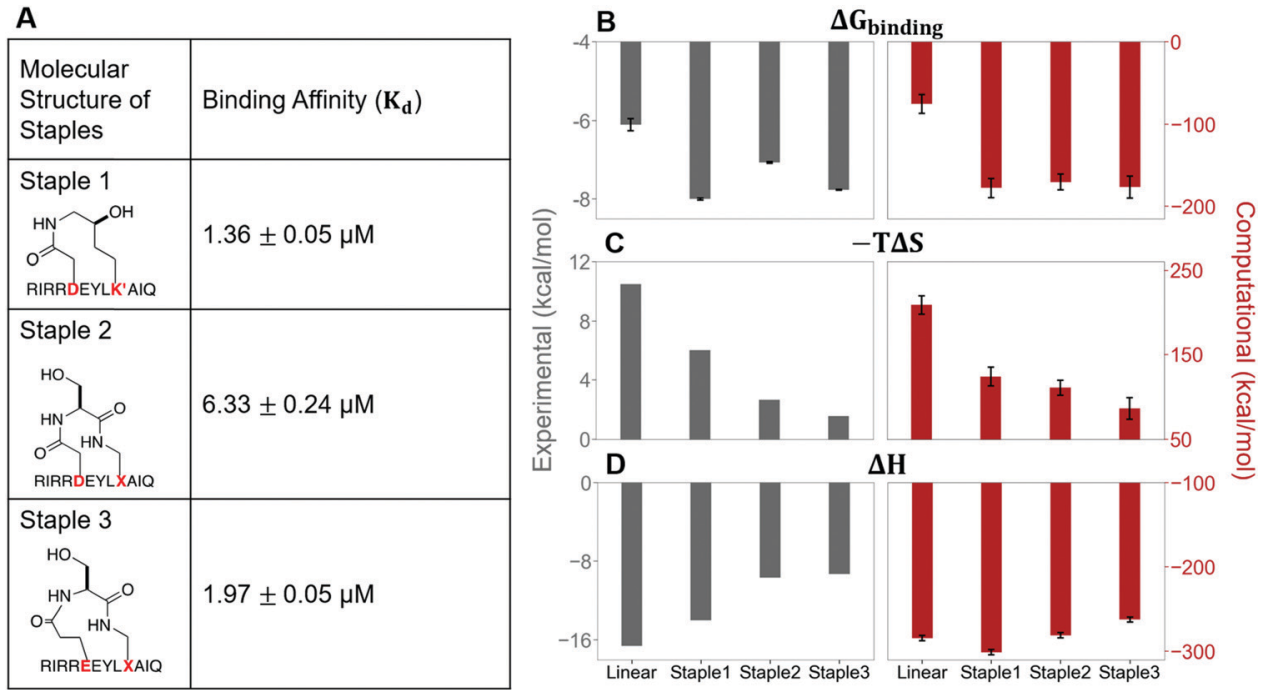

Fig. 8 The molecular structures of three stapled peptides, staple 1 , staple 2 and staple 3 along with the binding affinity, $K_{\mathrm{d}}$ (Left). Thermodynamic properties of GK binding with linear, staple 1, staple 2, and staple 3 peptides. (A) Binding free energy $\left(\Delta G_{\text {binding }}\right)$, (B) entropy difference ( $\left.-T \Delta S\right)$, and (C) enthalpy difference $(\Delta H)$ as measured by ITC (gray bar and left figure) and calculated by MMPBSA and Quasi-harmonic approximation (red bar and right figure). 


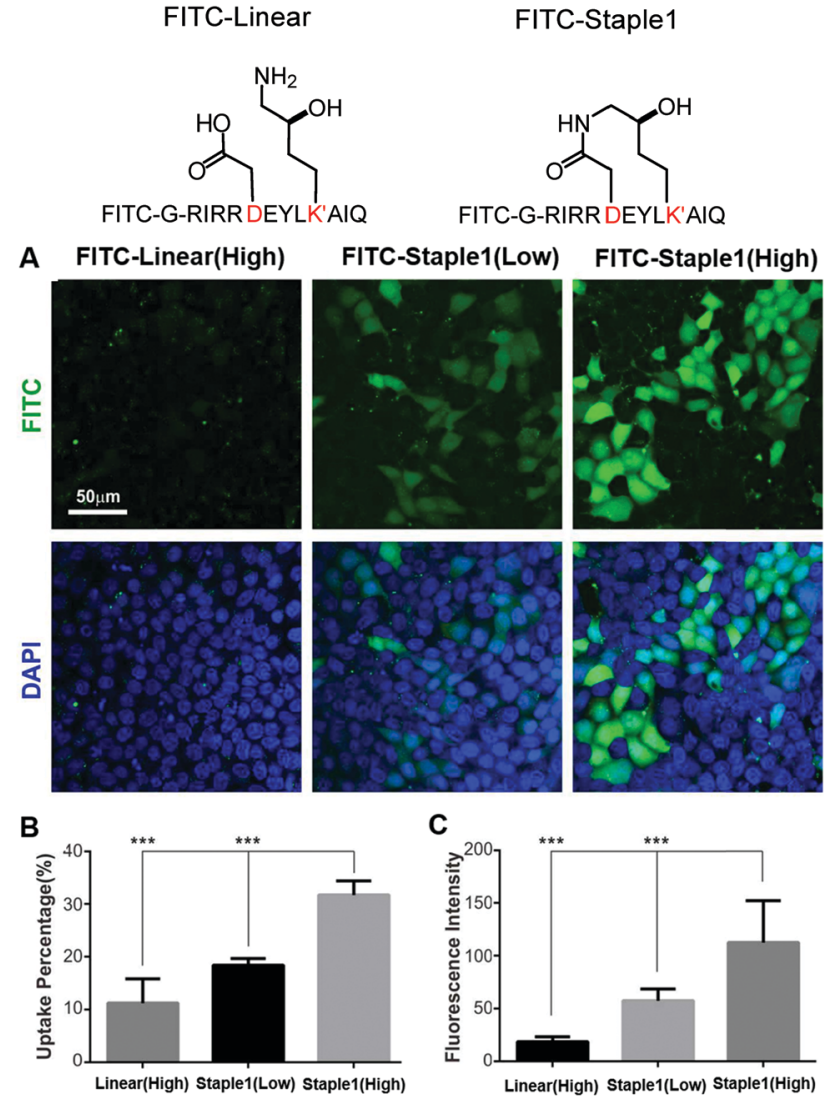

Fig. 9 Stapling facilitates peptide cellular penetration. (A) Cellular uptake of linear or staple 1 peptides in MDCK cells. Cells were treated with FITC labeled linear or stapled peptides (at $10 \mu \mathrm{M} /$ low or $20 \mu \mathrm{M} /$ high concentrations) dissolved in culture media. (B) Cell uptake percentage of FITClabeled peptides. For a fixed region, the cell uptake percentage is defined by cells with FITC signals divided by the total cell amount. Data were presented as mean \pm SEM, $n=3$. (C) FITC intensity within the cell. The average intensity of all cells with FITC signals in a fixed region was calculated. Data were presented as mean $\pm \mathrm{SEM}, n=3$

calculations show that $\Delta H$ is less favorable for the binding of staple 2 and 3 peptides compared to the staple 1 peptide (Fig. 8C and D). As a result, the overall binding affinity is comparable for all three stapled peptides (Fig. 8). These results indicate that the stapled peptide design that increases the entropy of the bound state ( $\left.S_{\text {bound }}\right)$ may not be effective due to the energy-entropy compensation.

\section{Stapled peptide penetrates the cell better than linear peptide}

In this section, we further examined the cell penetrability of our novel staple using the staple 1 peptide. As shown in Fig. 9, the staple 1 peptide displays substantially better cell penetrability than the linear peptide. The cell penetrability of linear and staple 1 peptides was analyzed by treating MDCK cells with FITC-labelled peptides at $10 \mu \mathrm{M}$ (low) or $20 \mu \mathrm{M}$ (high) concentrations (calculated as the peptide concentration in culture media). There is a significantly higher percentage of uptake of the Staple 1 peptide by MDCK cells than the linear peptide (Fig. 9B). The average intensity of FITC in MDCK cells is also higher for the staple 1 peptide compared to the linear peptide, indicating that the staple 1 peptide can more effectively penetrate the cell membrane at a given concentration (e.g. $20 \mu \mathrm{M}$, Fig. 9C). This shows that our designed staple 1 peptide can indeed penetrate living cells with a high efficiency, indicating that this stapled peptide may be a promising tool to study PSD-95 and SAPAP interaction in vivo.

In addition to stapled peptide designs that benefit from entropy, we expect that the binding affinity for the GK-domain inhibitors could also be improved by enhancing the interaction energies in the future. One promising strategy is to consider replacing the glutamic acid in our stapled peptides with nonnatural amino acid with additional negative charges, which can further enhance its interactions with the phosphor-binding pocket because glutamic acid may not be sufficient to mimic the salt bridge between phosphoserine and the conserved binding site (e.g. residue R568 and R571 of the GK domain in Fig. S4, ESI $\dagger$ ). For example, one could use a non-hydrolysable phosphoserine analog to further enhance the binding interactions. $^{24}$

\section{Conclusions}

In this study, we successfully designed a stapled peptide (staple 1), which can bind to the PSD-95 GK domain with a 25 -fold stronger binding affinity than its linear counterpart. To ensure good solubility of the stapled peptide, we employed a recently developed synthetic strategy to introduce hydrophilic groups into the staple. ${ }^{25}$ We also showed that the novel staple has better cell penetrability than the linear peptide, indicating its future applications for use in in vivo study. More importantly, we were able to quantitatively dissect the enthalpic and entropic contributions of binding affinity of peptide ligands to the GK domain, and further correlate molecular features with specific thermodynamics terms. We found that the stronger binding affinity of the staple 1 peptide is mainly due to the staple that constrained the peptide conformation in the free state, leading to a smaller absolute entropy of ligand in the free state $\left(\left(S_{\text {free }}\right)_{\text {igand }}\right)$. Based on staple 1, we further explored two other stapled peptides (staple 2 and 3), which exerted even larger entropy gains due to more flexible bound states $\left(\left(S_{\text {bound }}\right)_{\text {protein }}\right)$. Surprisingly, both staple 2 and 3 peptides display binding affinity comparable with that of the staple 1 peptide, due to the energy-entropy compensation of the bound state. Our results suggest that increasing the stability of the stapled peptide in free solution is an effective strategy for the rational design of stapled peptide inhibitors.

\section{Methods}

\section{Protein expression and purification}

Expression and purification of human or rat PSD-95 GK have been described previously. ${ }^{18,19,26}$ 


\section{Peptide synthesis}

A. Fmoc-based solid phase peptide synthesis (SPPS). Rink amide-AM resin (100 mg) was swollen in dry $\mathrm{CH}_{2} \mathrm{Cl}_{2}(3 \mathrm{~mL})$ for 10 minutes and then washed with $\mathrm{CH}_{2} \mathrm{Cl}_{2}(6 \times 3 \mathrm{~mL})$. The removal of the Fmoc group was executed using a deblock solution of $20 \%$ piperidine in DMF at room temperature for $20 \mathrm{~min}$. The resin was washed with $\mathrm{DMF}(5 \times 3 \mathrm{~mL}), \mathrm{CH}_{2} \mathrm{Cl}_{2}$ $(5 \times 3 \mathrm{~mL})$, and DMF $(5 \times 3 \mathrm{~mL})$. The resin was subsequently submitted to iterative peptide assembly (Fmoc-SPPS). The following Fmoc amino acids were employed: Fmoc-Ala-OH, Fmoc-Arg(Pbf)-OH, Fmoc-Glu( $t \mathrm{Bu})-\mathrm{OH}, \quad$ Fmoc-Gln(Trt)-OH, Fmoc-Hyl(Boc)(Psi(Me, Me)pro)-OH, Fmoc-Ile-OH, Fmoc-Leu$\mathrm{OH}$ and Fmoc-Tyr( $t \mathrm{Bu})-\mathrm{OH}$. Fmoc-AspBenzofuran-OH, FmocGluBenzofuran-OH and Fmoc-Dap-Ser-OH dipeptide were obtained by synthesis (ESI $\dagger$ ). For the coupling step, a solution of Fmoc-AA-OH (4 equiv. according to the resin capacity), HATU (4 equiv. relative to resin capacity) and DIEA (8 equiv. relative to resin capacity) in DMF was added and the resin was shaken at room temperature for 1 hour. The resin was washed with DMF $(5 \times 3 \mathrm{~mL})$ and $\mathrm{CH}_{2} \mathrm{Cl}_{2}(5 \times 3 \mathrm{~mL})$.

B. Full deprotection to obtain crude free peptides from resin with cocktail A. A mixture solution of TFA/ $\mathrm{H}_{2} \mathrm{O} / \mathrm{TIPS}(95 \% / 2.5 \%$ / $2.5 \%$, cocktail A) was added to the resin bound peptide, and the mixture was gently agitated for $2 \mathrm{~h}$ at room temperature. The resin was then washed with $\mathrm{CH}_{2} \mathrm{Cl}_{2}(5 \mathrm{~mL} \times 6)$. The solvent was blown off under a stream of condensed air. The crude product was triturated with cold diethyl ether to give a white suspension, which was centrifuged and the ether was subsequently decanted.
C. Ozonolysis of the crude peptide to form SAL ester. The crude peptide obtained from general procedure $\mathrm{B}$ was dissolved in the mixture solvent of $\mathrm{H}_{2} \mathrm{O} / \mathrm{ACN}=5: 1$ with $5 \%$ TFA in an ice bath. The solution was treated with $\mathrm{O}_{3}$, which was produced from an ozone generator, for $1 \mathrm{~min}$. The resulting solution was diluted with $\mathrm{H}_{2} \mathrm{O}$ and ready for HPLC purification with a separation yield of $12-18 \%$ based on the loading of the resin.

D. Intramolecular STL to form the stapled peptides. The peptide obtained from general procedure $\mathrm{C}$ was dissolved in the solvent of pyridine/HOAc $\left(1: 2, \mathrm{~mol} \mathrm{~mol}^{-1}\right)$ and the concentration of the peptide was adjusted to $0.5 \mathrm{mM}$. The resulting solution was stirred at room temperature for about $6 \mathrm{~h}$ and monitored by LC-MS. No dimer was formed but the product, and the solvent was blown off under a stream of condensed air, followed by treatment with the cocktail of TFA $/ \mathrm{H}_{2} \mathrm{O} / \mathrm{TIPS}=95 \%$ / $2.5 \% / 2.5 \%$ for about $10 \mathrm{~min}$. After precipitation with cold diethyl ether, the resulting peptide residue was ready for HPLC purification with a separation yield of $35-60 \%$.

\section{CD spectrum}

CD samples were dissolved in HPLC grade $\mathrm{H}_{2} \mathrm{O}$ at $200 \mu \mathrm{M}$ concentration. CD spectra were acquired on a Jasco J-815 circular dichroism (CD) spectropolarimeter. Spectra were processed using OriginLab.

\section{Isothermal titration calorimetry assay}

Isothermal titration calorimetry experiments were carried out on a MicroCal ITC200 calorimeter (Malvern) at $25{ }^{\circ} \mathrm{C}$.

Table 1 Data collection and refinement statistics of DLG GK/inhibitory peptides

\begin{tabular}{|c|c|c|}
\hline Dataset & GK/linear peptide & GK/stapled peptide \\
\hline \multicolumn{3}{|l|}{ Data collection } \\
\hline Space group & $p 21$ & $p 1$ \\
\hline Unit cell $(a, b, c, \AA)$ & $35.854,61.579,103.851$ & $51.029,51.954,144.5$ \\
\hline Unit cell $\left(\alpha, \beta, \gamma,{ }^{\circ}\right)$ & $90.000,98.29,90.000$ & $91.775,91.429,120.965$ \\
\hline Wavelength (̊) & 0.97774 & 0.97799 \\
\hline \multirow[t]{2}{*}{ Resolution range $(\AA)$} & $50.00-2.45$ & $50.00-2.60$ \\
\hline & $(2.49-2.45)$ & $(2.64-2.60)$ \\
\hline No. of unique reflections & $15628(777)$ & 37969 (1893) \\
\hline Redundancy & $4.1(4.0)$ & $3.8(3.7)$ \\
\hline$I / \sigma$ & $8.5(1.8)$ & $9.9(1.9)$ \\
\hline Completeness (\%) & $93.9(96.2)$ & $97.2(97.1)$ \\
\hline$R_{\text {merge }}(\%)^{a}$ & $13.9(41.3)$ & $14.7(69.4)$ \\
\hline \multicolumn{3}{|l|}{ Structure refinement } \\
\hline Resolution, $\AA$ & $35.14-2.45(2.53-2.45)$ & $33.14-2.60(2.69-2.60)$ \\
\hline$R_{\text {work }}^{b} / R_{\text {free }}^{c}(\%)$ & $0.2094(0.2755) / 0.2706(0.3731)$ & $0.2199(0.2908) / 0.2684(0.3660)$ \\
\hline Rmsd bonds/angles $\left(\AA{ }^{\circ}\right)$ & $0.008 / 1.07$ & $0.008 / 1.22$ \\
\hline Average $B$ factor $\left(\AA^{2}\right)$ & 37.98 & 48.9 \\
\hline No. of protein atoms & 3210 & 9424 \\
\hline No. of water molecules & 198 & 382 \\
\hline \multicolumn{3}{|l|}{ No. of reflections } \\
\hline Working set & $14819(1426)$ & $36046(3402)$ \\
\hline Test set & $792(77)$ & $1906(203)$ \\
\hline \multicolumn{3}{|l|}{ Ramachandran plot (\%) } \\
\hline Favored regions & 96.6 & 96.4 \\
\hline Allowed regions & 3.4 & 3.4 \\
\hline Outliers & 0 & 0.17 \\
\hline
\end{tabular}

Numbers in parentheses represent the value for the highest resolution shell. ${ }^{a} R_{\text {merge }}=\sum\left|I_{\mathrm{i}}-I_{\mathrm{m}}\right| / \sum I_{\mathrm{i}}$, where Ii is the intensity of the measured reflection and $I_{\mathrm{m}}$ is the mean intensity of all symmetry related reflections. ${ }^{b} R_{\text {cryst }}=\sum\left\|F_{\text {obs }}|-| F_{\text {calc }}\right\| / \sum\left|F_{\text {obs }}\right|$, where $F_{\text {obs }}$ and $F_{\text {calc }}$ are observed and calculated structure factors. ${ }^{c} R_{\text {free }}=\sum T|| F_{\text {obs }}|-| F_{\text {calc }} \| / \sum T\left|F_{\text {obs }}\right|$, where $T$ is a test data set of about $5 \%$ of the total reflections randomly chosen and set aside prior to refinement. 
The concentration of the injected samples (peptides) in the syringe was $200 \mu \mathrm{M}$, and the concentration of the samples in the cell was fixed at $20 \mu \mathrm{M}$. The sample in the syringe was sequentially injected into the sample cell with a time interval of $150 \mathrm{~s}(0.5 \mu \mathrm{L}$ for the first injection and $2 \mu \mathrm{L}$ each for the following 19 injections). The titration data were analyzed using the Origin 7.0 software and fitted with the one-site binding model.

\section{Crystallization, data collection and processing}

Both crystals of rat DLG4 GK in complex with the synthesized peptide were obtained by the sitting drop vapor diffusion method at $16{ }^{\circ} \mathrm{C}$. Freshly purified DLG4 GK protein was concentrated to $10 \mathrm{mg} \mathrm{ml}^{-1}$ before the addition of the synthesized peptide in a $2: 1$ molar ratio.

The GK/linear-peptide complex crystal was grown in solution containing (0.02 M citric acid, $0.08 \mathrm{M}$ BIS-TRIS propane/ pH 8.8), 16\% w/v polyethylene glycol 3350. Glycerol (30\%) was added as the cryo-protectant before the crystal was frozen in liquid nitrogen. The GK/stapled-peptide complex crystal was grown in solution containing 35\% w/v PEP 629 and $100 \mathrm{mM}$ HEPES ( $\mathrm{pH}$ 7.5). This kind of crystal was frozen directly in liquid nitrogen. A $2.45 \AA$ resolution X-ray data set for the GK/linear-peptide and a $2.60 \AA$ resolution X-ray data set for the GK/stapled-peptide were collected at the beam-line BL19U1 or BL17U1 of the Shanghai Synchrotron Radiation Facility. The diffraction data sets were processed and scaled by HKL3000.

The data qualities were checked using phenix.xtriage, and pseudo-merohedral twinning was found in both data sets. Using the structure of the DLG1 GK domain (PDB id: 3UAT) as the search model, the initial structural models were solved using the molecular replacement method in PHASER for both GK/ linear-peptide and GK/stapled-peptide complexes. The models were then refined by the phenix.refinement. Coot was used for peptide modelling and model adjustments. TLS refinement was applied at the final refinement stage. For the GK/linear-peptide complex, the data quality was largely improved by applying twin refinement (twin_fraction $=0.09$, twin_law $=h,-k,-h,-1)$. For the GK/Stapled-peptide complex, the model was also improved by applying twin refinement (twin_fraction $=0.07$, twin_law $=-\mathrm{k}, \mathrm{h}, \mathrm{-l}$ ). The final structure was validated by phenix.model_vs_data validation tools. The final refinement statistics are listed in Table 1. The structure figures were prepared using the program VMD Molecular Graphic Viewer ${ }^{27}$ (http://www.ks.uiuc.edu/ Research/vmd/). PDB id for the linear peptide/GK complex: 7F7G, PDB id for the stapled peptide/GK complex: 7F7I.

\section{NMR spectrum}

$1 \mathrm{D}{ }^{1} \mathrm{H}$ water suppression NMR was performed in $90 \% \mathrm{H}_{2} \mathrm{O}$ and $10 \% \mathrm{D}_{2} \mathrm{O}$. NMR spectra were acquired on a Bruker Avance DRX 500 FT-NMR spectrometer at $298 \mathrm{~K}$ and processed using TopSpin software.

\section{Cell culture and peptide cellular uptake}

MDCK cells were cultured on $35 \mathrm{~mm}$ dishes with $10 \mathrm{~mm}$ diameter uncoated glass bottom (MatTek) in DMEM (Hyclone,
SH30022.01B) supplemented with 10\% fetal bovine serum (FBS; Hyclone, SH30084.03) and $50 \mu \mathrm{M}$ penicillin-streptomycin. They were cultured in a $37{ }^{\circ} \mathrm{C}$ incubator with $5 \% \mathrm{CO}_{2}$ for around 3 days after seeding.

For the peptide cellular uptake experiment, the cells were rinsed three times with PBS to completely remove the medium. Peptides were dissolved in PBS with 1\% glucose and diluted using DMEM with no FBS. Concentrations of the peptides were determined by measuring A280 and A488 using a NanoDrop spectrophotometer (Thermo Fisher Scientific). The cells were then applied with one milliliter of the medium containing $10 \mu \mathrm{M}$ or $20 \mu \mathrm{M}$ peptides at $37{ }^{\circ} \mathrm{C}$ for 4 hours. After the incubation, the cells were fixed using 4\% PFA for the imaging.

\section{Microscopy and data analysis}

All the cell culture images were acquired on a Zeiss LSM 880 laser-scanning confocal microscope. Images were captured using a $40 \times 1.4$ oil objective with pinhole setting to 1 Airy unit. The obtained images were then analyzed with ImageJ software (http://imagej.nih.gov/ij/).

\section{Assignment of partial charges of staple peptides and hydroxylysine for MD simulation}

As the stapled peptides and the linear peptide contain nonconventional amino acids, we performed geometry optimization and electrostatic potential calculation for each type of staple peptide (staple 1, staple 2, and staple 3) and hydroxylysine. For each type of staple peptide, we performed geometry optimization of the peptide with sequence, ACE-AXEYLXA-NME, where $\mathrm{Xs}$ are the stapled residues, ACE is acetyl to cap the N-terminal and NME is $N$-methyl to cap the C-terminal. For hydroxylysine, the amino acid is capped by ACE and NME. For these modelled structures, geometry optimizations were performed using Gaussian $09^{28}$ with the Becke, 3-parameter, Lee-Yang-Parr method and basis set $6-31 g^{*}$ (b3lyp/6-31G*). Electrostatic potential calculations were performed using the Hartree-Fock method with basis set $6-31 \mathrm{~g}^{*}\left(\mathrm{hf} / 6-31 \mathrm{~g}^{*}\right)$. The partial charges were then calculated by the RESP method using resp modules in AmberTools 13, ${ }^{29}$ where partial charges were determined by a two-stage $\mathrm{RESP}^{30}$ procedure. In the first stage, partial charges were fitted to the electrostatic potential while maintaining the partial charges of ACE, NME, Alanine, and -EYL- sequence, the same as AMBER 99SB-ILDN. In addition, the partial charges of $\mathrm{N}-\mathrm{H}$ and $\mathrm{C}=\mathrm{O}$ groups of the stapled residues (X) were maintained to be the same as the non-charged residues in AMBER 99SB-ILDN. At the second stage, fitting was done for methylene and methyl groups while maintaining the partial charges of all the other atoms.

\section{Molecular dynamics simulation of the GK-ligand complex}

The crystal structures of the GK-linear complex and GK-staple 1 complex were used as the initial structures of Molecular Dynamics (MD) simulation. The complexes of GK-staple 2 and GK-staple 3 were modelled based on the GK-staple 1 crystal structure. 
All complexes were solvated in a dodecahedron box with TIP3P ${ }^{31}$ water molecules. To neutralize the system, $\mathrm{Na}^{+}$and $\mathrm{Cl}^{-}$ ions were added to $0.15 \mathrm{~mol}$ per liter salt concentration. We performed all MD simulations using the GROMACS $4.5^{32}$ simulation package and the AMBER 99SB-ILDN ${ }^{33}$ force field along with the partial charges obtained by the RESP method for staple and non-conventional amino acids. Long-range electrostatic interactions were treated by the Particle-Mesh Ewald (PME) method $^{34}$ and van der Waals short-range interactions were calculated using a cut-off of $10 \AA$.

Energy minimization was performed using the steepest descent method, followed by $N V T$ equilibration for $1 \mathrm{~ns}$ with position restraints on the heavy atoms at $300 \mathrm{~K}$ using a V-rescale thermostat ${ }^{35}$ with a coupling constant of $0.1 \mathrm{ps.} \mathrm{At}$ this step, the positions of heavy atoms of the GK-ligand complex were restrained with a force constant of $1000 \mathrm{~kJ} \mathrm{~mol}^{-1}$ $\mathrm{nm}^{-1}$ and all bonds in protein molecules and water were constrained using the LINCS $^{36}$ algorithm. NPT equilibration was then carried out for 1 ns using the Parrinello-Rahman barostat $^{37}$ with a coupling constant $=0.2 \mathrm{ps}$ to a reference pressure of 1 bar with position restraints on the heavy atoms of the GK-ligand complex using $1000 \mathrm{~kJ} \mathrm{~mol}^{-1} \mathrm{~nm}^{-1}$ force constant. The restraints were released by $200 \mathrm{~kJ} \mathrm{~mol}^{-1} \mathrm{~nm}^{-1}$ every 200 ps. Lastly, the position restraints were released and MD simulation was run for 400 ps under NPT conditions.

For each complex, four production MD simulations under NPT conditions were performed for $200 \mathrm{~ns}$. In the succeeding MD simulations, we used the same simulation package, force field, non-bonded interaction calculation method, barostat, thermostat, and bond constraint. For the following analysis, the first $25 \mathrm{~ns}$ was removed from each simulation.

\section{Molecular dynamics simulation of the GK domain}

The starting structure for MD simulations was taken from the crystal structure of the GK-staple 1 complex with staple 1 removed. It was solvated in a dodecahedron box with 8797 TIP3P water molecules. The system also contains $30 \mathrm{Na}^{+}$ions and $27 \mathrm{Cl}^{-}$ions to neutralize the system and creates a $0.15 \mathrm{~mol}$ per liter salt environment. Energy minimization was performed using the steepest descent method, followed by NVT equilibration for $100 \mathrm{ps}$ with position restraints (force constant = $1000 \mathrm{~kJ} \mathrm{~mol}^{-1} \mathrm{~nm}^{-1}$ ) on the heavy atom of the GK domain at $T=300 \mathrm{~K}$ with coupling constant $=0.1 \mathrm{ps} . N P T$ equilibration was then carried out for $100 \mathrm{ps}$ with coupling constant $=0.2 \mathrm{ps}$ to a reference pressure of 1 bar with position restraints on the heavy atoms of the GK domain. Four production NPT MD simulations were performed for $200 \mathrm{~ns}$ each. For the following analysis, the first $25 \mathrm{~ns}$ was removed from each simulation.

\section{Molecular dynamics simulation of peptide ligands}

The starting structure for MD simulation was obtained from the GK-staple 1 complex for staple 1, staple 2, and staple 3 peptides and the GK-linear complex for the linear peptide, with the GK domain removed. It was solvated in a dodecahedron box with TIP3P water molecules, $\mathrm{Na}^{+}$ions and $\mathrm{Cl}^{-}$ions to neutralize the system and creates a $0.15 \mathrm{~mol}$ per liter salt environment.
Energy minimization was performed using the steepest descent method, followed by NVT equilibration for 100 ps with position restraints (force constant $=1000 \mathrm{~kJ} \mathrm{~mol}^{-1} \mathrm{~nm}^{-1}$ ) on the heavy atom of ligands at $T=300 \mathrm{~K}$ with coupling constant $=0.1 \mathrm{ps}$. NPT equilibration was then carried out for $100 \mathrm{ps}$ with coupling constant $=0.2 \mathrm{ps}$ to a reference pressure of 1 bar. Four production NPT MD simulations were performed for $200 \mathrm{~ns}$ each. For the following analysis, the first 25 ns was removed from each simulation.

\section{Computational thermodynamic property calculation}

Binding free energy can be written as $\Delta G_{\text {binding }}=G_{\text {complex }}-$ $G_{\text {ligand }}-G_{\text {protein }}$. The free energies of the complex, protein and ligand ( $G_{\text {complex }}, G_{\text {ligand }}$, and $G_{\text {protein }}$ ) were approximated by calculation of the solvation free energy, solute potential energy in a vacuum, and solute entropy, $G_{\mathrm{x}}=G_{\text {solvation }}+E_{\mathrm{MM}}-T S$, using the structures from the complex, protein and ligand MD simulations, respectively. $G_{\text {solvation }}$ was calculated by solving the linear Poisson-Boltzmann equation for the polar contribution $\left(G_{\text {polar }}\right)$ and the solvent accessible surface area equation for the non-polar $\left(G_{\text {non-polar }}\right)$ contribution, $G_{\text {solvation }}=G_{\text {polar }}+G_{\text {non-polar }}$, using MM/PBSA implementation in GROMACS (g_mmpbsa, ${ }^{38,39}$ ). The box for $G_{\text {polar }}$ calculation was created by using the extreme coordinates of the complex in each dimension, then expanded it by 1.5 -fold at each direction $(\mathrm{cfac}=1.5)$. The ionic strength of the system is $0.15 \mathrm{M} \mathrm{NaCl}$ with $0.95 \AA$ and $1.81 \AA$ radii of $\mathrm{Na}^{+}$and $\mathrm{Cl}^{-}$, respectively. Vacuum, solute and solvent dielectric constants were set as 1, 1, and 80, respectively. Non-polar solvation energy was calculated using the solvent accessible surface area method, with probe radius $=1.4 \AA, \gamma=0.022678 \mathrm{~kJ} \mathrm{~mol}^{-1} \AA^{-2}$, and offset $=$ $3.84982 \mathrm{~kJ} \mathrm{~mol}^{-1} \AA^{-2}$.

The potential energies of the protein, ligand, and complex in a vacuum, $E_{\mathrm{MM}}$, were calculated by GROMACS. The Coulomb interaction was calculated between all the atoms in each system using Coulomb's law. The potential energy consists of a bonded angle and dihedral energy, coulomb short range and 1-4, as well as Lennard-Jones short range and 1-4 interactions.

For each frame in the MD simulation of the complex, protein and ligand, $G_{\text {solvation }}+E_{\mathrm{MM}}$ was calculated. Then, to obtain the average and error bar, we combined all four simulations and just maintained the values at every 100th step, totalling 1400 values. Next, we did bootstrap of the 1400 values with 1400 samples. For each bootstrap sample, the mean was calculated. Then, the reported average is the average of 1400 bootstrap mean values and the error bar is the standard deviation from 1400 bootstrap mean values. Finally, the energy change, $\Delta G_{\text {solvation }}+$ $\Delta E_{\mathrm{MM}}$, was obtained by subtracting the values from complex simulation (bound state) from the values from the protein and ligand simulation (free state). The values of $\Delta G_{\text {solvation }}+\Delta E_{\mathrm{MM}}$ are compared with experimental $\Delta \mathrm{H}$ measured by ITC shown in Fig. 5 .

The conformational entropy, $\Delta S$, was calculated by subtracting the absolute entropy of the protein/ligand in bound form from that of the protein/ligand in the free state, $\Delta S=\left(S_{\text {bound }}-\right.$ $\left.S_{\text {free }}\right)_{\text {protein }}+\left(S_{\text {bound }}-S_{\text {free }}\right)_{\text {ligand }}$. The absolute entropy was calculated by quasi-harmonic approximation ${ }^{40}$ with Cartesian coordinates, using g_covar and g_analyze modules in GROMACS. 
All the structures in MD simulation were first fitted to all $\mathrm{C} \alpha$ atoms of the GK domain and $\mathrm{C} \alpha$ of residues -1 to 3 of peptide ligands for calculation of entropy of the GK domain and peptide ligand, respectively. For each system (GK-ligand complex, GK, or peptide ligands), we performed four $200 \mathrm{~ns}$ simulations and calculated the absolute entropy $(S)$ of the GK and ligand from each simulation. The $S_{\text {bound }}$ value of the GK and ligand was calculated from the structures of the GK and ligand obtained by MD simulations of the GK-ligand complex, respectively. The $S_{\text {free }}$ value of the GK and ligand was calculated from the structures of the GK and ligand obtained by the MD simulation of the GK or ligand in solution, respectively. Thus, there are $4 \times 4$ combinations to subtract the absolute entropy of the bound state $\left(S_{\text {bound }}\right)$ from that of the free state $\left(S_{\text {free }}\right)$ for the GK and ligand system,

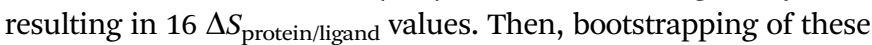
16 values was performed 800 times to calculate the average and error bar. The reported average in Fig. 5 is the addition of bootstrap average of entropy difference $(\Delta S)$ of the protein and ligand, $\Delta S_{\text {ligand }}+\Delta S_{\text {protein, }}$ and the error bar is obtained by error propagation of standard deviation of bootstrap samples of $\Delta S_{\text {protein }}$ and $\Delta S_{\text {ligand }}$.

\section{Contribution}

ICU and SC conducted the computation, JX and CHPC conducted the chemical synthesis, and YS, RZ, XC and PPHC conducted the biological experiment and structural biology. $\mathrm{DB}, \mathrm{MZ}, \mathrm{XH}$ and $\mathrm{XL}$ initiated and supervised the project. ICU, CHPC, YS, XH and XL wrote the manuscript with inputs from all authors.

\section{Conflicts of interest}

There are no conflicts to declare.

\section{Acknowledgements}

We thank the National Facility for Protein Science Shanghai (NFPS) for allowing us to use the BL19U1 beamline and the Shanghai Synchrotron Radiation Facility (SSRF) for X-ray beam time (BL17U1 beamline). This work was supported by the Hong Kong Research Grant Council (17309616, T13-605/18-W, AoE/ M-09/12 and AoE/P-705/16), Innovation and Technology Commission (ITCPD/17-9), and a grant from the Asia Cancer Foundation. X. H. acknowledges the Padma Harilela Associate Professorship. MZ is a Kerry Holdings Professor in Science and a Senior Fellow of IAS at HKUST.

\section{References}

1 J. A. Wells and C. L. McClendon, Reaching for high-hanging fruit in drug discovery at protein-protein interfaces, Nature, 2007, 450(7172), 1001-1009.
2 P. Vlieghe, V. Lisowski, J. Martinez and M. Khrestchatisky, Synthetic therapeutic peptides: science and market, Drug DiscoveryToday, 2010, 15(1-2), 40-56.

3 T. A. Hill, N. E. Shepherd, F. Diness and D. P. Fairlie, Constraining cyclic peptides to mimic protein structure motifs, Angew. Chem., Int. Ed., 2014, 53(48), 13020-13041.

4 R. S. Harrison, et al., Novel helix-constrained nociceptin derivatives are potent agonists and antagonists of ERK phosphorylation and thermal analgesia in mice, J. Med. Chem., 2010, 53(23), 8400-8408.

5 Y. H. Lau, et al., (2015) Double Strain-Promoted Macrocyclization for the Rapid Selection of Cell-Active Stapled Peptides. Angew. Chem., Int. Ed. 54(51):15410-3.

6 R. S. Harrison, et al., Downsizing human, bacterial, and viral proteins to short water-stable alpha helices that maintain biological potency, Proc. Natl. Acad. Sci. U. S. A., 2010, 107(26), 11686-11691.

7 T. K. Sawyer, et al., Macrocyclic $\alpha$ helical peptide therapeutic modality: A perspective of learnings and challenges, Bioorgan. Med. Chem., 2018, 26(10), 2807-2815.

8 Y. S. Tan, D. P. Lane and C. S. Verma, Stapled peptide design: principles and roles of computation, Drug Discovery Today, 2016, 21(10), 1642-1653.

9 J. A. Miles, et al., Hydrocarbon constrained peptides understanding preorganisation and binding affini, Chem. Sci., 2016, 7, 3694-3702.

$10 \mathrm{~J}$. Zhu, Y. Shang and M. Zhang, Mechanistic basis of MAGUK-organized complexes in synaptic development and signalling, Nat. Rev. Neurosci., 2016, 17(4), 209-223.

11 E. Kim, et al., GKAP, a novel synaptic protein that interacts with the guanylate kinase- like domain of the PSD-95/SAP90 family of channel clustering molecules, J. Cell Biol., 1997, 136(3), 669-678.

12 S. Naisbitt, et al., Characterization of guanylate kinase-associated protein, a postsynaptic density protein at excitatory synapses that interacts directly with postsynaptic density-95/synapse-associated protein 90, J. Neurosci., 1997, 17(15), 5687-5696.

13 M. Takeuchi, et al., SAPAPs. A family of PSD-95/SAP90associated proteins localized at postsynaptic density, J. Biol. Chem., 1997, 272(18), 11943-11951.

14 S. G. N. Grant, Synaptopathies: Diseases of the synaptome, Curr. Opin. Neurobiol., 2012, 22(3), 522-529.

15 O. Marin, Interneuron dysfunction in psychiatric disorders, Nat. Rev. Neurosci., 2012, 13(2), 107-120.

16 P. Monteiro and G. Feng, SHANK proteins: Roles at the synapse and in autism spectrum disorder, Nat. Rev. Neurosci., 2017, 18(3), 147-157.

17 T. Bourgeron, From the genetic architecture to synaptic plasticity in autism spectrum disorder, Nat. Rev. Neurosci., 2015, 16(9), 551-563.

$18 \mathrm{~J}$. Zhu, et al., Guanylate kinase domains of the MAGUK family scaffold proteins as specific phospho-proteinbinding modules, EMBO J., 2011, 30(24), 4986-4997.

$19 \mathrm{~J}$. Zhu, et al., Phosphorylation-dependent interaction between tumor suppressors Dlg and Lgl, Cell Res., 2014, 24(4), 451-463. 
20 C. A. Johnston, C. Q. Doe and K. E. Prehoda, Structure of an enzyme-derived phosphoprotein recognition domain, PLoS One, 2012, 7(4), e36014.

21 K. Hirao, et al., A novel multiple PDZ domain-containing molecule interacting with $\mathrm{N}$ - methyl-D-aspartate receptors and neuronal cell adhesion proteins, J. Biol. Chem., 1998, 273(33), 21105-21110.

22 J. Zhu, et al., Synaptic Targeting and Function of SAPAPs Mediated by Phosphorylation-Dependent Binding to PSD-95 MAGUKs, Cell Rep., 2017, 21(13), 3781-3793.

23 D. J. Dunican and P. Doherty, Designing cell-permeant phosphopeptides to modulate intracellular signaling pathways, Biopolym. - Pept. Sci. Sect., 2001, 60(1), 45-60.

24 G. Ye, et al., Synthesis and evaluation of tripodal peptide analogues for cellular delivery of phosphopeptides, J. Med. Chem., 2007, 50(15), 3604-3617.

25 C. H. P. Cheung, et al., Construction of diverse peptide structure architectures via chemoselective peptide ligation, Chem. Sci., 2021, 12(20), 7091-7097.

26 Y. Xia, Y. Shang, R. Zhang and J. Zhu, Structure of PSD-95/ MAP1A complex reveals unique target recognition mode of MAGUK GK domain, Biochem. J., 2017, 474(16), 2817-2828.

27 W. Humphrey, A. Dalke and K. Schulten, VMD: Visual molecular dynamics, J. Mol. Graphics, 1996, 14(1), 33-38.

28 M. J. Frisch, et al., Gaussian 09 A.02, Gaussian, Inc, Wallingford CT, 2009, DOI: 111.

29 D. A. Case, et al., AmberTools 13, Univ California, San Fr, 2013.

30 W. D. Cornell, P. Cieplak, C. I. Bayly, P. A. Kollman and P. A. Kollmann, Application of RESP Charges To Calculate Conformational Energies, Hydrogen Bond Energies, and Free Energies of Solvation, J. Am. Chem. Soc., 1993, 115(7), 9620-9631.
31 W. L. Jorgensen, J. Chandrasekhar, J. D. Madura, R. W. Impey and M. L. Klein, Comparison of simple potential functions for simulating liquid water, J. Chem. Phys., 1983, 79(2), 926-935.

32 S. Pronk, et al., GROMACS 4.5: A high-throughput and highly parallel open source molecular simulation toolkit, Bioinformatics, 2013, 29(7), 845-854.

33 K. Lindorff-Larsen, et al., Improved side-chain torsion potentials for the Amber ff99SB protein force field, Proteins: Struct., Funct. Bioinf., 2010, 78(8), 1950-1958.

34 T. Darden, D. York and L. Pedersen, Particle mesh Ewald: An $\mathrm{N} \cdot \log (\mathrm{N})$ method for Ewald sums in large systems, J. Chem. Phys., 1993, 98(12), 10089.

35 G. Bussi, D. Donadio and M. Parrinello, Canonical sampling through velocity rescaling, J. Chem. Phys., 2007, 126(1), 014101.

36 B. Hess, H. Bekker, H. J. C. Berendsen and J. G. E. M. Fraaije, LINCS: A Linear Constraint Solver for molecular simulations, J. Comput. Chem., 1997, 18(12), 1463-1472.

37 M. Parrinello and A. Rahman, Polymorphic transitions in single crystals: A new molecular dynamics method, J. Appl. Phys., 1981, 52(12), 7182-7190.

38 R. Kumari, R. Kumar and A. Lynn, G-mmpbsa -A GROMACS tool for high-throughput MM-PBSA calculations, J. Chem. Inf. Model., 2014, 54(7), 1951-1962.

39 N. A. Baker, D. Sept, S. Joseph, M. J. Holst and J. A. McCammon, Electrostatics of nanosystems: Application to microtubules and the ribosome, Proc. Natl. Acad. Sci. U. S. A., 2001, 98(18), 10037-10041.

40 M. Karplus and J. N. Kushick, Method for Estimating the Configurational Entropy of Macromolecules, Macromolecules, 1981, 14(2), 325-332. 